\title{
RISK MINIMIZING STRATEGIES FOR A PORTFOLIO OF INTEREST-RATE SECURITIES
}

\author{
ANDRZEJ PALCZEWSKI \\ Institute of Applied Mathematics \\ University of Warsaw \\ Banacha 2, 02-097 Warszawa, Poland \\ E-mail: apalczew@mimuw.edu.pl
}

\begin{abstract}
The paper presents an application of stochastic control methods to fixed income management in an incomplete market with external economic factors. The objective of an investor is the minimization of a shortfall risk. The problem is reduced to the multidimensional Bellman equation. It is shown that for a large class of loss functions the equation possesses a continuous solution. We also consider loss functions from the HARA class and prove that for such functions the Hamilton-Jacobi-Bellman equation has a sufficiently smooth solution. This solution guarantees the existence of a well defined investment strategy. A special example of the bond portfolio with interest rates governed by the Gaussian HJM model is solved explicitly.
\end{abstract}

1. Introduction. Application of stochastic control to portfolio management has a long tradition. It started about 30 years ago with the works of Merton [19], [20] and has been developed by numerous authors (see the books of Karatzas and Shreve [15] and Korn [16] for references). In the majority of analyzed problems stochastic control has been used to manage the portfolio of equities. There is a limited number of papers when fixed income assets are included in the portfolio. Bonds appear in the portfolio usually in the context of the bond-stock mix. That is the case of Brennan, Schwartz and Lagnado [6] and Brennan and Xia [7], where the bond-stock-cash mix has been considered. Their main technical tool was the Hamilton-Jacobi-Bellman PDE, which was solved numerically or reduced to an ordinary differential equation by imposing a particular form of the utility function.

2000 Mathematics Subject Classification: Primary 93E20; Secondary 91B28.

Key words and phrases: optimal portfolios, asset-liability management, stochastic interest rate.

This work was partially supported by The Polish Committee of Scientific Research under grant PBZ KBN 016/P03/99.

The paper is in final form and no version of it will be published elsewhere. 
One can find very few examples when stochastic control methods have been used solely to fixed income management. The reason is well explained in the paper by Bielecki and Pliska [3] who have written: "First, most fixed income assets have finite lives, so they cannot be modeled by simple stochastic processes such as the infinitely-lived geometric Brownian motions that are commonly used for equities. (...) Another possible explanation $(\ldots)$ is that, unlike equities, the risk and return characteristics of fixed income assets explicitly depend upon underlying exogenous factors, namely, levels of interest rates. Consequently, sensible trading strategies will need to be explicit functions of these interest rates and so these interest rate factors need to be explicitly incorporated in any mathematical model."

Explicit results on fixed income management have been obtained by Sørensen [22], Deelstra, Grasselli and Koehl [12] and Bajeux-Besnainou, Jordan and Portait [1]. They have solved a bond management problem by the martingale solution technique advanced by Cox and Huang [9], [10] for some special models of interest rates (Vasicek or CIR). The results are restricted to the complete market only.

Bielecki and Pliska [3] applying risk sensitive criterion to the management of fixed income portfolio have avoided the problem with finite maturity time by using the concept of rolling-horizon bonds introduced by Rutkowski [21]. Recently Bielecki, Pliska and Yong [4] have solved the management problem for the class of HARA utility functions and the model of interest rates proposed by Duffie and Kan [13]. The solution of the Hamilton-Jacobi-Bellman equation was also the main technical tool used by Liu [17] who solved a problem very similar to the problem approached in this paper. Being unable to solve the resulting PDE in full generality, Liu restricted his solution to a special class of quadratic diffusion processes.

The problem we analyze is the asset-liability management. To describe what we understand by the asset-liability management let us consider the portfolio of obligations (liabilities). These obligations can be treated, without loss of generality, as zero-coupon bonds with given maturities. The aim is to find the investment portfolio of zero-coupon bonds (assets) which hedge the obligations in an optimal way.

To solve this problem we have to supplement it with additional constraints. First, we restrict the class of accessible investment assets. As asset and liabilities are zero-coupon bonds, allowing in the investment portfolio bonds of same maturities as bonds in the liability portfolio, we obtain a trivial solution. Namely, investing in the bonds with the same maturities as the bonds in the liability portfolio we obtain perfect hedging. When we restrict the investment to the bonds with the maturities different from the maturities of the bonds in the liability portfolio, the liability portfolio became non-liquid. Then the portfolio problem has to be considered in the framework of an incomplete market and we cannot expect perfect hedging of the liabilities. In the paper we analyze this incomplete market situation.

Then we have to select a cost functional in our problem. Since perfect hedging is impossible and super-hedging is usually very expensive, we have chosen as the objective the minimization of a shortfall risk. The shortfall risk as an investment objective has been introduced by Föllmer and Leukert [14] for complete and incomplete markets with 
securities described by continuous semimartingales. It is defined as

$$
\mathbb{E}\left(g\left(H-V_{T}\right)^{+}\right),
$$

where $H$ is a contingent claim, $V_{T}$ a hedging portfolio at time $T$ and $g(x)$ a loss function, which is convex for a risk averse investor.

The essential difficulty in applying this approach to the asset-liability management of fixed income assets is the dependence of the portfolio wealth on external factors (short interest rate at least). This leads to the multidimensional Hamilton-Jacobi-Bellman equation. Any assumption on the special form of the loss function cannot reduce the differential problem to an ODE. We have to deal with a highly nonlinear partial differential equation. The first step is the proof of existence of sufficiently smooth solutions to this equation (this problem has been completely neglected in [6]). The essential part of the present paper is to show that the problem is well-posed and under additional assumptions on the loss function has a solution which is sufficiently smooth to guarantee the existence of a well defined investment strategy.

The plan of the paper is as follows. In Section 2, we present the portfolio problem arising from the asset-liability management. In this section we prove also that the HJB equation arising from the control problem possesses a generalized (viscosity) solution. In Section 3, we show that under additional assumptions on the loss function we can find a smooth solution to the HJB equation and construct effectively an optimal investment strategy. Section 4 is devoted to the special case in which the economic factors are reduced to the HJM model of the spot interest rate. In this case we obtain the explicit solution of the portfolio problem. Section 5 analyzes the same model in the case when the liability matures at the planning horizon. This problem has been carefully analyzed by Bielecki, Pliska and Yong [4]. Here we present another solution of the problem.

2. The portfolio problem. We consider an asset and liability management problem, where the prices of securities (zero coupon bonds) are functions of external state variables (economic factors). The security prices satisfy the following equations

$$
\frac{d P\left(t, T_{j}\right)}{P\left(t, T_{j}\right)}=\mu_{j}(t, R) d t+\sum_{i=1}^{d} \sigma_{i j}(t, R) d W_{i}(t), \quad j=1,2, \ldots, N
$$

where $\mu_{j}$ and $\sigma_{i j}$ depend on an $M$-dimensional vector $R$ of economic factors, which follow the Markovian diffusion process

$$
d R_{m}(t)=\mu_{m}^{r}(t, R) d t+\sum_{i=1}^{d} b_{i m}(t, R) d W_{i}(t), \quad m=1,2, \ldots, M .
$$

Assumption 2.1. For the model of bond prices we assume that the coefficients $\mu_{j}(t, R)$ and $\sigma_{i j}(t, R)$ are bounded and continuous for $t \in[0, T]$ and $R \in \mathbb{R}^{M}$.

For the model of economic factors we make typical assumptions which guarantee the existence of strong solutions to equation (2), i.e. we assume that $\mu_{m}^{r}(t, R)$ and $b_{i m}(t, R)$ are continuous functions of their arguments, which in addition fulfil the estimates

$$
\begin{aligned}
& \left|\mu_{m}^{r}\left(t, R_{1}\right)-\mu_{m}^{r}\left(t, R_{2}\right)\right|+\left|b_{i m}\left(t, R_{1}\right)-b_{i m}\left(t, R_{2}\right)\right| \leq c\left|R_{1}-R_{2}\right|, \\
& \left|\mu_{m}^{r}(t, R)\right|^{2}+\left|b_{i m}(t, R)\right|^{2} \leq c^{2}\left(1+|R|^{2}\right),
\end{aligned}
$$

for $t \in[0, T], R, R_{1}, R_{2} \in \mathbb{R}^{M}$, where $c$ is a positive constant. 
Let us observe that we assume the log-normality of bond prices but the stochastic model of economic factors is quite general. Later we shall make some additional assumptions to guarantee well-posedness of the problem.

In the rest of the paper we consider bonds with maturities $T_{j}$ from a fixed set of maturities $\left\{T_{1}, T_{2}, \ldots, T_{N}\right\}$. The liabilities are bonds due at dates $T_{l}$, where $T_{l}$ belong to a subset of $\left\{T_{1}, T_{2}, \ldots, T_{N}\right\}$. To simplify the notation let $K:=\{1, \ldots, N\}$. We say that the bond with maturity $T_{l}$ is a liability when $l \in L$, where $L$ is a fixed subset of $K$. We assume also that the liabilities are known, i.e. their amounts are known and deterministic. The liability portfolio $H$ consists of $\eta_{l}^{\star}$ bonds maturing at time $T_{l}(l \in L)$. The value at time $t$ of the liability portfolio is given by

$$
H(t):=\sum_{l \in L} \eta_{l}^{\star} P\left(t, T_{l}\right) .
$$

We hedge this liability portfolio at time $T \leq T_{k}$, for all $k \in K$, with the asset portfolio of bonds with maturities $T_{j}$ for $j \in K \backslash L$. Consider now the asset portfolio $\Pi$ consisting of $\pi_{j}^{\star}$ bonds maturing at date $T_{j}, j \in K \backslash L$. The value of the asset portfolio equals

$$
\Pi^{\pi}(t):=\sum_{j \in K \backslash L} \pi_{j}^{\star} P\left(t, T_{j}\right) .
$$

As it has been observed by Björk [5], when the dimensionality of random factors (in our case the dimensionality $d$ of the Wiener process $W(t))$ is the same as the dimensionality of the bond portfolio used for hedging then the market is complete. Since we are interested in the situation in which the liabilities cannot be perfectly hedged with the assets, we assume that the dimensionality $d$ of the Wiener process is much larger than the number $N$ of bonds with different maturities. In such a case the market is incomplete and perfect hedging is not possible.

We optimize the portfolio of assets and liabilities with respect to the cost of the shortfall at time $T$ with the given initial endowment $x$ and the initial state of economic factors $r$. Let $V^{\pi}(t):=H(t)-\Pi^{\pi}(t)$ be the value of the portfolio. The cost of the shortfall is measured by the terminal cost function $g: \mathbb{R} \rightarrow \mathbb{R}$. Therefore, the optimization problem is to minimize the expected value of $g\left(V^{\pi}(T)\right)$ conditioned on the initial endowment $x \in \mathbb{R}$ and the initial state of economic factors $r \in \mathbb{R}^{M}$. The portfolio evolution is given by the equation (we omit the index $\pi$ )

$$
\begin{aligned}
d V(t)= & \sum_{l \in L} \eta_{l}^{\star} d P_{l}-\sum_{j \notin L} \pi_{j}^{\star} d P_{j}=\sum_{l \in L} \eta_{l}^{\star}\left\{\mu_{l} d t+\sum_{i=1}^{d} \sigma_{i l} d W_{i}(t)\right\} P\left(t, T_{l}\right) \\
& -\sum_{j \notin L} \pi_{j}\left\{\mu_{j} d t+\sum_{i=1}^{d} \sigma_{i j} d W_{i}(t)\right\} P\left(t, T_{j}\right) \\
= & \sum_{l \in L} \eta_{l}^{\star} \mu_{l} P\left(t, T_{l}\right) d t+\sum_{i=1}^{d} \sum_{l \in L} \eta_{l}^{\star} \sigma_{i l} P\left(t, T_{l}\right) d W_{i}(t) \\
& -\sum_{j \notin L} \pi_{j} V(t) \mu_{j} d t-\sum_{i=1}^{d} \sum_{j \notin L} \pi_{j} \sigma_{i j} V(t) d W_{i}(t),
\end{aligned}
$$


where $\pi_{j}:=\pi_{j}^{\star} P\left(t, T_{j}\right) / V$ is the fraction of the total wealth $V(t)$ invested in the bond with maturity $T_{j}$.

To define the control problem in a more rigorous way we have to specify the set of admissible strategies $\pi$ and the equation for the evolution of the state variables $\left(V^{\pi}(t), R(t), P\left(t, T_{l}\right): l \in L\right)$.

Definition 2.1. The set of admissible strategies $S^{\pi}$ is given by the conditions:

1. $\pi:[0, T] \times \Omega \rightarrow U \subseteq \mathbb{R}_{+}^{N}$ is measurable, bounded and $\left\{\mathcal{F}_{t}\right\}_{t \geq 0}$-adapted,

2. $V^{\pi}(0)=x$ (budget constraint).

The portfolio equation can be written in the more compact form

$$
d V(t)=\sum_{l \in L}\left(\mu^{l} d t+\sum_{i=1}^{d} \Lambda_{i l} d W_{i}(t)\right) P\left(t, T_{l}\right)-\left(\mu^{V} d t+\sum_{i=1}^{d} \theta_{i} d W_{i}(t)\right) V(t),
$$

where

$$
\mu^{V}=\sum_{j \notin L} \pi_{j} \mu_{j}, \quad \theta_{i}=\sum_{j \notin L} \pi_{j} \sigma_{i j}, \mu^{l}=\eta_{l}^{\star} \mu_{l}, \quad \Lambda_{i l}=\eta_{l}^{\star} \sigma_{i l} .
$$

2.1. The value function and the HJB equation. To solve the optimal portfolio problem we use the technique of dynamical programming. Therefore, we consider the family of optimal portfolio problems parameterized by the initial values of the state variables $\left(V^{\pi}(t), R_{m}(t), P\left(t, T_{l}\right): l \in L\right)$

$$
\min _{\pi \in S^{\pi}\left(t, x, r_{m}, p_{l}: l \in L\right)} \mathbb{E}\left[g\left(V^{\pi}(T)\right) \mid V^{\pi}(t)=x ; R_{m}(t)=r_{m} ; P\left(t, T_{l}\right)=p_{l}: l \in L\right] .
$$

Let us introduce the value function for these problems

$$
u\left(t, x, r_{m}, p_{l}: l \in L\right):=\min _{\pi \in S^{\pi}} \mathbb{E}\left[g\left(V^{\pi}(T)\right) \mid V^{\pi}(t)=x ; R_{m}(t)=r_{m} ; P\left(t, T_{l}\right)=p_{l}: l \in L\right] .
$$

The state variables $\left(V(t), R_{m}(t), P\left(t, T_{l}\right): l \in L\right)$ satisfy equations (7), (2) and (1), respectively. The control is given by $\left\{\pi_{j}(t): j \notin L\right\}$ and is hidden in $\mu^{V}$ and $\theta_{i}$.

Let us now introduce some additional assumptions.

Assumption 2.2. $U$ is a complete, separable metric space and $0<T<\infty$.

Assumption 2.3. The loss function $g(x)$ is locally Lipschitz continuous.

Under Assumptions 2.1, 2.2 and 2.3 equation (7) admits a unique solution and the value function is well defined.

Next, the existence of the optimal strategy will be considered. Let us recall some auxiliary results from the stochastic control theory (cf. [23]).

LemMA 2.1. Under Assumptions 2.2 and 2.3 the value function $u(s, y)$, where $y$ stands for the variables $\left(x, r_{m}, p_{l}: l \in L\right)$, satisfies the inequalities

$$
|u(s, y)| \leq K(1+|y|) \quad \forall_{s \in[0, T], y \in \mathbb{R}^{1+M+\sharp L}}
$$

and

$$
|u(s, y)-u(\hat{s}, \hat{y})| \leq K\left(|y-\hat{y}|+(1+|y| \vee|\hat{y}|)|s-\hat{s}|^{\frac{1}{2}}\right) \quad \forall_{s, \hat{s} \in[0, T], y, \hat{y} \in \mathbb{R}^{1+M+\sharp L}} .
$$


Lemma 2.2. If Assumptions 2.1, 2.2 and 2.3 are satisfied and the value function $u$ is $C^{1}$ with respect to $t$ and $C^{2}$ with respect to all other variables, then it is a solution of the Hamilton-Jacobi-Bellman equation

$$
\begin{aligned}
& u_{t}+\frac{1}{2} \sum_{k \in L} \sum_{l \in L} u_{p_{k} p_{l}} S_{k l} p_{k} p_{l}+\sum_{m=1}^{M} \sum_{k \in L} u_{r_{m} p_{k}} p_{k} \sum_{i=1}^{d} b_{i m} \sigma_{i k}+\frac{1}{2} \sum_{m=1}^{M} \sum_{n=1}^{M} u_{r_{m} r_{n}} \sum_{i=1}^{d} b_{i m} b_{i n} \\
& +\sum_{k \in L} u_{p_{k}} p_{k} \mu_{k}+\sum_{m=1}^{M} u_{r_{m}} \mu_{m}^{r}+u_{x} \sum_{k \in L} \mu^{k} p_{k}+\sum_{k \in L} u_{x p_{k}} p_{k} \sum_{l \in L} \sum_{i=1}^{d} \Lambda_{i l} \sigma_{i k} p_{l} \\
& \quad+\sum_{m=1}^{M} u_{x r_{m}} \sum_{k \in L} p_{k} \sum_{i=1}^{d} b_{i m} \Lambda_{i k}+\frac{1}{2} u_{x x} \sum_{k \in L} \sum_{l \in L} \sum_{i=1}^{d} \Lambda_{i k} \Lambda_{i l} p_{k} p_{l}+\inf _{\pi \in S^{\pi}}\left(\frac{1}{2} u_{x x} x^{2} \sum_{i=1}^{d} \theta_{i}^{2}\right. \\
& \left.\quad-x \sum_{k \in L} u_{x p_{k}} p_{k} \sum_{i=1}^{d} \sigma_{i k} \theta_{i}-u_{x x} x \sum_{k \in L} p_{k} \sum_{i=1}^{d} \Lambda_{i k} \theta_{i}-u_{x} \mu^{V} x-\sum_{m=1}^{M} u_{x r_{m}} x \sum_{i=1}^{d} \theta_{i} b_{i m}\right)=0,
\end{aligned}
$$

where $S_{k l}=\sum_{i=1}^{d} \sigma_{i k} \sigma_{i l}$, with the terminal condition $u\left(T, x, r_{m}, p_{l}: l \in L\right)=g(x)$.

THEOREM 2.1. Let us assume 2.1, 2.2 and 2.3 and let $\left(x, r_{m}, p_{l}: l \in L\right)$ be in a compact set of $\mathbb{R} \times \mathbb{R}^{M} \times \mathbb{R}^{\sharp L}$ ( $\sharp$ denotes cardinality). Then the HJB equation (9) possesses a unique continuous viscosity solution $u\left(t, x, r, p_{l}: l \in L\right)$.

From Theorem 2.1 it follows that the HJB equation (9) possesses a unique viscosity solution (the value function). On the other hand, it is well known (cf. [23]) that if the value function $u\left(t, x, r_{m}, p_{l}: l \in L\right)$ is of class $C^{1}$ with respect to $t$ and $C^{2}$ with respect to other variables, then it is the classical solution of the HJB equation (9). When $u\left(t, x, r_{m}, p_{l}\right.$ : $l \in L)$ is a smooth function then we arrive easily at the optimal strategy

$$
\begin{aligned}
\pi_{j}=\left(u_{x x} x\right)^{-1} \sum_{h \notin L}\left(S^{-1}\right)_{j h}\left(u_{x} \mu_{h}+u_{x x} \sum_{k \in L} \sum_{i=1}^{d} \Lambda_{i k} p_{k} \sigma_{i h}\right. & +\sum_{m=1}^{M} u_{x r_{m}} \sum_{i=1}^{d} b_{i m} \sigma_{i h} \\
& \left.+\sum_{k \in L} u_{x p_{k}} p_{k} \sum_{i=1}^{d} \sigma_{i k} \sigma_{i h}\right),
\end{aligned}
$$

for all $j \in K \backslash L$.

Hence to find an optimal strategy we have to know that the solution of the HJB equation is from the class $C^{1,2}$. General theory (Theorem 2.1) gives only continuity of the value function and the optimal strategy given by formula (10) is not well defined. Since the HJB equation is a nonlinear PDE, to prove that its solutions are smooth is a real challenge. This problem can be solved for some particular classes of nonlinear equations, cf. the book by Ladyzhenskaya, Solonnikov and Uraltseva [18], where this problem is discussed for quasilinear equations, or the paper [2], where the problem is solved for semilinear equations in the context of hedging and valuation of contingent claims. The above formulated equation (9) is a so called fully nonlinear equation and its smooth solutions can be obtained only under additional assumptions. 
3. The smooth value function. As we have observed in the previous section, to explore the strong point of dynamic programming approach, i.e. explicit formulae for investment strategies, we need a smooth solution to the HJB equation. Since in general, we get only a continuous viscosity solution, we have to impose additional assumptions to obtain smooth solutions. To this end we have to simplify our problem. We neglect the fact that the liability portfolio consists of zero-coupon bonds and treat this portfolio as a log-normal stochastic process $H(t)$ which fulfils the equation

$$
d H(t):=H(t)\left(\mu^{H} d t+\sum_{i=1}^{d} \xi_{i} d W_{i}(t)\right) .
$$

Let us observe that this dynamics of $H(t)$ is obtained in particular when the portfolio $H$ consists of a single bond.

The portfolio of assets $V(t)$ is

$$
V^{\pi}(t):=\sum_{j \in K} \pi_{j}^{\star} P\left(t, T_{j}\right),
$$

where bonds of all maturities can be put in this portfolio (but we still assume that the liability is nonhedgeable by the asset portfolio).

Denoting by $\pi_{j}$ the fraction of the total wealth $V(t)$ invested in the bond with maturity $T_{j}$, i.e. $\pi_{j}=\pi_{j}^{\star} P\left(t, T_{j}\right) / V$, we can write

$$
d V^{\pi}(t)=V^{\pi}(t)\left(\mu^{V} d t+\sum_{i=1}^{d} \theta_{i}^{V} d W_{i}(t)\right),
$$

where $\mu^{V}=\sum_{j \in K} \pi_{j} \mu_{j}$ and $\theta_{i}^{V}=\sum_{j \in K} \pi_{j} \sigma_{i j}$.

Then we define the new process

$$
X(t)=\frac{H(t)}{V^{\pi}(t)}
$$

and obtain the following equation for its evolution

$$
\frac{d X}{X}=\mu^{X} d t+\sum_{i=1}^{d} \theta_{i} d W_{i}
$$

where

$$
\theta_{i}=\xi_{i}-\theta_{i}^{V}, \quad \mu^{X}=\mu^{H}-\mu^{V}-\sum_{i=1}^{d} \theta_{i}^{V} \theta_{i} .
$$

We optimize the portfolio $X(t)$ with respect to the vector of strategies $\pi=\left(\pi_{1}, \ldots, \pi_{N}\right)$. That is a different optimization problem: we optimize the quotient liabilities over assets. This approach enables us to consider losses and gains in the same framework. Such an approach is not quite new in the financial literature. It has been applied by Browne [8] to analyze optimal portfolios which exceed the performance of a given stochastic benchmark. In that case Browne has analyzed as the state variable the ratio of the investor's portfolio to the benchmark. A similar quotient has been used by Dai Pra, Runggaldier and Tolotti [11] who have optimized the quadratic loss in the benchmark tracking problem.

The admissible strategies for our problem are defined as follows. 
DEFInition 3.1. The set of admissible strategies $S^{\pi}$ is given by the conditions:

1. $\pi:[0, T] \times \Omega \rightarrow U \subseteq \mathbb{R}^{N}$ is measurable, bounded and $\left\{\mathcal{F}_{t}\right\}_{t \geq 0}$-adapted,

2. $X(0)=x$ (budget constraint).

The optimization problem is now of the form

$$
\min _{\pi \in S^{\pi}\left(t, x, r_{m}\right)} \mathbb{E}\left[g(X(T)) \mid X(t)=x, R_{m}(t)=r_{m}\right] .
$$

Introducing the value function

$$
u\left(t, x, r_{m}\right):=\min _{\pi \in S^{\pi}\left(t, x, r_{m}\right)} \mathbb{E}\left[g(X(T)) \mid X(t)=x, R_{m}(t)=r_{m}\right],
$$

we arrive at the following Hamilton-Jacobi-Bellman equation

$$
\begin{aligned}
& u_{t}+\inf _{\pi \in S^{\pi}}\left(x u_{x} \mu^{X}+\sum_{m=1}^{M} u_{r_{m}} \mu_{m}^{r}+\frac{1}{2} x^{2} u_{x x} \sum_{i=1}^{d} \theta_{i}^{2}\right. \\
& \left.+\frac{1}{2} \sum_{m=1}^{M} \sum_{n=1}^{M} u_{r_{m} r_{n}} \sum_{i=1}^{d} b_{i m} b_{i n}+x \sum_{m=1}^{M} u_{r_{m} x} \sum_{i=1}^{d} b_{i m} \theta_{i}\right)=0, \\
& u(T, x, r)=g(x) .
\end{aligned}
$$

To obtain smooth solutions to the HJB equation we make the following assumption.

Assumption 3.1. The loss function $g(x)$ is from the generalized HARA class and is given by the expression $g(x)=c x^{\alpha}$, where $\alpha \neq-1$ and $x \in(-\infty, \infty)$.

Under the above assumption we postulate that the value function can be factorized in the form

$$
u(t, x, r)=g(x) q(t, r)
$$

Substituting the above expression into equation (15) we obtain

$$
\begin{aligned}
& q_{t}+\inf _{\pi \in S^{\pi}}\left(\alpha q \mu^{X}+\sum_{m=1}^{M} q_{r_{m}} \mu_{m}^{r}+\frac{\alpha(\alpha-1)}{2} q \sum_{i=1}^{d} \theta_{i}^{2}\right. \\
& \left.\quad+\frac{1}{2} \sum_{m=1}^{M} \sum_{n=1}^{M} q_{r_{m} r_{n}} \sum_{i=1}^{d} b_{i m} b_{i n}+\alpha \sum_{m=1}^{M} q_{r_{m}} \sum_{i=1}^{d} b_{i m} \theta_{i}\right)=0 \\
& q(T, r)=1 .
\end{aligned}
$$

From this equation we can calculate formally the optimal investment strategy

$$
\pi_{j}=\frac{1}{1+\alpha} \sum_{h \in K} S_{j h}^{-1}\left(\mu_{h}+\alpha Z_{h}+\sum_{m=1}^{M} \frac{q_{r_{m}}}{q} B_{m h}\right),
$$


where

$$
\begin{aligned}
& S_{j h}=\sum_{i=1}^{d} \sigma_{i j} \sigma_{i h}, \quad j, h \in K, \\
& Z_{h}=\sum_{i=1}^{d} \xi_{i} \sigma_{i h}, \quad h \in K, \\
& B_{m h}=\sum_{i=1}^{d} b_{i m} \sigma_{i h}, \quad h \in K .
\end{aligned}
$$

The optimal strategy can be written in the abbreviated form

$$
\pi_{j}=\pi_{j}^{0}+\sum_{m=1}^{M} \frac{q_{r_{m}}}{q} \pi_{j m}^{1},
$$

where

$$
\begin{aligned}
& \pi_{j}^{0}=\frac{1}{1+\alpha} \sum_{h \in K} S_{j h}^{-1}\left(\mu_{h}+\alpha Z_{h}\right), \\
& \pi_{j m}^{1}=\frac{1}{1+\alpha} \sum_{h \in K} S_{j h}^{-1} B_{m h} .
\end{aligned}
$$

Substituting expression (19) into the HJB equation (17) we obtain

$$
\begin{aligned}
q_{t}+\alpha q \bar{\mu}^{X} & +\sum_{m=1}^{M} q_{r_{m}} \mu_{m}^{r}+\frac{\alpha(\alpha-1)}{2} q \sum_{i=1}^{d} \bar{\theta}_{i}^{2}+ \\
& +\frac{1}{2} \sum_{m=1}^{M} \sum_{n=1}^{M} q_{r_{m} r_{n}} \sum_{i=1}^{d} b_{i m} b_{i n}+\alpha \sum_{m=1}^{M} q_{r_{m}} \sum_{i=1}^{d} b_{i m} \bar{\theta}_{i}=0 .
\end{aligned}
$$

In the above equation the bar over $\theta_{i}$ and $\mu^{X}$ denotes the values of these parameters taken at the point of the optimal strategy. Using formula (19) we arrive at the expressions

$$
\begin{aligned}
& \bar{\mu}^{X}=a_{X}+\sum_{m=1}^{M} \frac{q_{r_{m}}}{q} b_{m X}+\sum_{m=1}^{M} \sum_{n=1}^{M} c_{m n} \frac{q_{r_{m}} q_{r_{n}}}{q^{2}} \\
& \sum_{i=1}^{d} \bar{\theta}_{i}^{2}=a_{\theta}+\sum_{m=1}^{M} b_{m \theta} \frac{q_{r_{m}}}{q}+\sum_{m=1}^{M} \sum_{n=1}^{M} c_{m n} \frac{q_{r_{m}} q_{r_{n}}}{q^{2}} \\
& \sum_{i=1}^{d} b_{i m} \bar{\theta}_{i}=a_{m b}+\sum_{n=1}^{M} b_{m n b} \frac{q_{r_{n}}}{q}
\end{aligned}
$$

where the coefficients are complicated functions of $\mu_{j}, \sigma_{i j}, b_{i m}$ and the optimal strategy:

$$
\begin{array}{rlrl}
a_{0} & =-2 \sum_{j \in K} \pi_{j}^{0} Z_{j}+\sum_{j \in K} \sum_{h \in K} \pi_{j}^{0} \pi_{h}^{0} S_{j h}, \quad b_{m}=\sum_{j \in K} \sum_{h \in K} \pi_{j}^{0} \pi_{m h}^{1} S_{j h}-\sum_{j \in K} \pi_{j m}^{1} Z_{j}, \\
c_{m n} & =\sum_{j \in K} \sum_{h \in K} \pi_{j m}^{1} \pi_{h n}^{1} S_{j h}, \\
a_{X} & =\mu^{H}+a_{0}-\sum_{j \in K} \pi_{j}^{0}\left(\mu_{j}-Z_{j}\right), & b_{m X} & =2 b_{m}-\sum_{j \in K} \pi_{j m}^{1}\left(\mu_{j}-Z_{j}\right),
\end{array}
$$




$$
\begin{aligned}
a_{\theta} & =a_{0}+\sum_{i=1}^{d} \xi_{i}^{2}, & b_{m \theta} & =2 b_{m}, \\
a_{m b} & =-\sum_{j \in K} \pi_{j}^{0} B_{j m}+\sum_{i=1}^{d} b_{i m} \xi_{i}, & b_{m n b} & =\sum_{j \in K} \pi_{j n}^{1} B_{j m} .
\end{aligned}
$$

After rearrangements the HJB equation (20) takes the form

$$
q_{t}+\sum_{m=1}^{M} \sum_{n=1}^{M} A_{0}^{m n} q_{r_{m} r_{n}}+\sum_{m=1}^{M} \sum_{n=1}^{M} A_{1}^{m n} \frac{q_{r_{m}} q_{r_{n}}}{q}+\sum_{m=1}^{M} A_{2}^{m} q_{r_{m}}+A_{3} q=0,
$$

where

$$
\begin{aligned}
A_{0}^{m n} & =\frac{1}{2} \sum_{i=1}^{d} b_{i m} b_{i n}, \\
A_{1}^{m n} & =\frac{1}{2} \alpha(\alpha+1) c_{m n}-\alpha b_{m n b}, \\
A_{2}^{m} & =\mu_{m}^{r}+\alpha b_{m X}+\frac{1}{2} \alpha(\alpha-1) b_{m \theta}+\alpha a_{m b}, \\
A_{3} & =\alpha a_{X}+\frac{1}{2} \alpha(\alpha-1) a_{\theta} .
\end{aligned}
$$

Equation (21) has to be solved in the strip $0 \leq t \leq T$ with the terminal condition

$$
q(T, r)=1 \text {. }
$$

Equation (21) is a quasilinear parabolic equation which possesses a solution provided this solution is bounded away from zero. To find this solution we make the substitution

$$
z=\ln q .
$$

For the new function $z$ we obtain the equation

$$
z_{t}+\sum_{m=1}^{M} \sum_{n=1}^{M} A_{0}^{m n} z_{r_{m} r_{n}}+\sum_{m=1}^{M} \sum_{n=1}^{M}\left(A_{0}^{m n}+A_{1}^{m n}\right) z_{r_{m}} z_{r_{n}}+\sum_{m=1}^{M} A_{2}^{m} z_{r_{m}}+A_{3}=0,
$$

with the terminal condition

$$
z(T, r)=0 .
$$

To solve equation (23) with condition (24) we use the well known theorem on the existence of smooth solutions to quasilinear parabolic equations (cf. [18]).

THEOREM 3.1. Let us consider an n-dimensional quasilinear parabolic equation

$$
u_{t}-\sum_{i, j=1}^{n} a_{i j}(t, x) u_{x_{i} x_{j}}+a\left(t, x, u, u_{x}\right)=0,
$$

in a bounded domain $\Omega_{T}=[0, T] \times \Omega$, where $\Omega$ is a bounded domain in $\mathbb{R}^{n}$ with boundary of class $H^{2+\beta}$. The above equation is considered with the boundary conditions

$$
u(t, x)=\psi(t, x), \quad \text { for }(t, x) \in \Gamma_{T},
$$

where $\Gamma_{T}=\partial \Omega \times[0, T] \cup \Omega \times\{t=0\}$. We assume that 
1. $\psi(t, x) \in H^{1+\beta / 2,2+\beta}\left(\Omega_{T}\right)$,

2. for $(t, x) \in \Omega_{T}$ we have $0<\nu \sum_{i=1}^{n} \xi_{i}^{2} \leq \sum_{i, j=1}^{n} a_{i j}(t, x) \xi_{i} \xi_{j} \leq \mu \sum_{i=1}^{n} \xi_{i}^{2}$ and $a(t, x, u, 0) u \geq-b_{1} u^{2}-b_{2}$ for every $u$ with $b_{1}, b_{2} \geq 0$,

3. for $(t, x) \in \Omega_{T},|u| \leq M$ and every $p$ the functions $a_{i j}(t, x)$ and $a(t, x, u, p)$ are continuous and $|a(t, x, u, p)| \leq \mu(1+|p|)^{2}$,

4. for $(t, x) \in \Omega_{T},|u| \leq M$ and $|p| \leq M$ the functions $a_{i j}(t, x)$ and $a(t, x, u, p)$ are Hölder continuous with respect to $t, x, u$ and $p$ with Hölder exponents $(\beta / 2, \beta, \beta, \beta)$,

5. $a(t, x, u, p)$ is differentiable with respect to $u$ and $p$, for $|u| \leq M$ and $|p| \leq M$, and we have the estimates

$$
\left|\frac{\partial a}{\partial p_{i}}\right| \leq \mu(M), \quad\left|\frac{\partial a}{\partial u}\right| \leq \mu(M) .
$$

Under the above assumptions there exists a unique solution to the considered boundary value problem in $H^{1+\beta / 2,2+\beta}\left(\Omega_{T}\right)$.

To apply Theorem 3.1 to equation (23) we have to make additional assumptions.

Assumption 3.2. Let $\mu_{j}, \mu^{H}, \xi_{i}$ and $\sigma_{i j}$ be Hölder continuous functions of $t$ with Hölder exponent $\beta / 2$, for some $\beta>0$.

Now we can prove our main theorem.

THEOREm 3.2. Under Assumptions 2.1, 2.2, 2.3, 3.1 and 3.2 there exists a unique solution $z(t, x)$ to the terminal problem (23)-(24) and $|z|,\left|z_{t}\right|,\left|z_{x_{i}}\right|,\left|z_{x_{i} x_{j}}\right|$ are bounded in $[0, T] \times \mathbb{R}^{M}$. This solution belongs to $H^{1+\beta / 2,2+\beta}\left(\Omega_{T}\right)$, where $\Omega_{T}=[0, T] \times \Omega$ and $\Omega$ is a bounded domain in $\mathbb{R}^{M}$.

Proof. Let us consider the terminal problem (23)-(24) in $\Omega_{T}=[0, T] \times \Omega$, where $\Omega$ is a fixed bounded domain in $\mathbb{R}^{M}$. To solve this problem we supplement equation (23) and terminal condition (24) with the boundary condition

$$
z(t, r)=0 \quad \text { for }(t, r) \in \partial \Omega \times[0, T] .
$$

The above defined augmented problem satisfies the assumptions of Theorem 3.1. In fact, the function $\psi(t, x)$ from Condition 1 in this theorem being equal to zero belongs to $H^{p, q}\left(\Omega_{T}\right)$ for arbitrary $p$ and $q$. The constants $\nu, \mu, b_{1}$ and $b_{2}$ from Condition 2 are independent of the chosen domain $\Omega$. The same is true about the constant $\mu$ from Condition 3 . Condition 4 is fulfilled due to Assumption 3.2. Condition 5 is fulfilled due to Assumption 2.2 and a simple quadratic form of the part of equation (23) which corresponds to the function $a\left(t, x, u, u_{x}\right)$ in Theorem 3.1.

Due to Theorem 3.1 there exists a unique solution of equation (23) with terminal condition (24) and boundary condition (25). This solution together with its derivatives can be estimated in $\Omega_{T}$ with constants which depend only on constants present in the assumptions of this theorem and not on the size of domain $\Omega$. Hence the solution which exists in any bounded domain $\Omega_{T}$ belongs to $H^{1+\beta / 2,2+\beta}\left(\Omega_{T}\right)$ and is uniformly bounded together with its derivatives independently of the size of the domain. Then we can consider a increased sequence of bounded smooth domains $\Omega^{n}$ that fill in the whole $\mathbb{R}^{M}$ and corresponding solutions $z^{n}$. By the standard Arzela-Ascoli theorem we can choose a 
subsequence of $z^{n}$ which converges to a function which is a solution to (23)-(24) on $[0, T] \times \mathbb{R}^{M}$.

REMARK 3.1. In many situations economic factors should be limited to nonnegative values only. In such cases Theorem 3.2 is still applicable as we can construct a sequence of bounded smooth domains $\Omega^{n}$ approximating the halfspace $\mathbb{R}_{+}^{M}$.

COROllary 3.1. Let us observe that due to Theorem 3.2 the solution $z(t, r)$ and its derivatives are bounded. Returning back to the original function $q$ we conclude that $q(t, r)$ is bounded away from zero and the quotient $q_{r_{m}} / q$ is bounded. It follows than that the optimal investment strategy given by expression (18) is bounded, i.e. admissible in accordance with Definition 3.1.

4. The portfolio problem in the Gaussian HJM model. In this section we present how our theory works in a simple case of a model with one dimensional set of economic factors. We assume that the economic factor is the spot interest rate and the prices of bonds are given by the Gaussian Heath-Jarrow-Morton model. In this model the instantaneously compounded forward rate satisfies the stochastic differential equation

$$
d f(t, T)=\alpha(t, T) d t+\tilde{\sigma}(t, T) d W(t) .
$$

We impose the following assumptions

1. $0<T \leq T^{*}$ is a fixed positive time, $t \in[0, T]$ and $T^{*}<+\infty$ is the finite time horizon,

2. $(W(t))_{t \in\left[0, T^{*}\right]}$ is the standard $d$-dimensional Brownian motion on the probability $\operatorname{space}\left(\Omega, \mathcal{F},\left(\mathcal{F}_{t}\right)_{t \in\left[0, T^{*}\right]}, \mathbb{P}\right)$,

3. $\int_{0}^{T}|\alpha(u, T)| d u+\int_{0}^{T}|\tilde{\sigma}(u, T)| d W(u)<\infty \quad \mathbb{P}$-a.s. for $T \in\left(0, T^{*}\right]$,

4. $\alpha$ and $\tilde{\sigma}$ are deterministic functions.

In the HJM framework the price at time $t$ of the zero-coupon bond maturing at some future date $T$ is

$$
P(t, T):=\exp \left(-\int_{t}^{T} f(t, u) d u\right) .
$$

It is well known that the price of the bond satisfies the equation

$$
d P(t, T)=P(t, T)\left(r(t) d t+\sigma(t, T) d W^{*}(t)\right)
$$

under measure $\mathbb{P}^{*}$, which is equivalent to $\mathbb{P}$ and under which the process $P(t, T) / B(t)$, the discounted bond price process, is a martingale. Here $B(t)=\exp \left(\int_{0}^{t} r(u) d u\right)$, with the $r(u)=f(u, u)$ - the instantaneous spot rate, $\left(W^{*}(t)\right)_{t \in[0, T]}$ is the Brownian motion with respect to $\mathbb{P}^{*}$ and $\sigma(t, T):=\int_{t}^{T} \tilde{\sigma}(t, u) d u$. Under measure $\mathbb{P}^{*}$ the forward rate $f(t, T)$ satisfies the equation

$$
d f(t, T)=\tilde{\sigma}(t, T) \sigma(t, T) d t+\tilde{\sigma}(t, T) d W^{*}(t) .
$$

We have also the following equation for the evolution of the spot rate $r(t)$

$$
d r(t)=a(t) d t+b(t) d W^{*}(t),
$$

where $a(t)=\left.\partial_{T} f(t, T)\right|_{T=t}$ and $b(t)=\tilde{\sigma}(t, t)$. 
We construct the optimal portfolio under the objective measure observed in the market. Therefore, we change the measure back to $\mathbb{P}$. This double change enables us to identify the market price of risk $\gamma_{i}(t)$ associated with the $i$-th coordinate of the $d$-dimensional Brownian motion $W$. Under measure $\mathbb{P}$ the price of the zero-coupon bond is a solution of the following equation

$$
\begin{aligned}
\frac{d P\left(t, T_{j}\right)}{P\left(t, T_{j}\right)} & =\left(r(t)+\sum_{i=1}^{d} \sigma_{i}\left(t, T_{j}\right) \gamma_{i}(t)\right) d t+\sum_{i=1}^{d} \sigma_{i}\left(t, T_{j}\right) d W_{i}(t) \\
& =\mu_{j}(t) d t+\sum_{i=1}^{d} \sigma_{i j}(t) d W_{i}(t),
\end{aligned}
$$

where $\mu_{j}:=r+\sum_{i=1}^{d} \sigma_{i}\left(t, T_{j}\right) \gamma_{i}(t)$ and $\sigma_{i j}(t)=\sigma_{i}\left(t, T_{j}\right)$.

The spot rate $r(t)$ under the market measure $\mathbb{P}$ satisfies the equation

$$
d r(t)=\left(a(t)+\sum_{i=1}^{d} \lambda_{i}(t) b_{i}(t)\right) d t+\sum_{i=1}^{d} b_{i}(t) d W_{i}(t)=\mu^{r} d t+\sum_{i=1}^{d} b_{i}(t) d W_{i}(t),
$$

where $\lambda_{i}(t)$ is the market price of risk for the spot rate associated with $W_{i}(t)$ and $\mu^{r}(t)=$ $a(t)+\sum_{i=1}^{d} \lambda_{i}(t) b_{i}(t)$.

The portfolio of liabilities $H(t)$ composed of the single bond with maturity $T_{0}$ fulfils the equation

$$
d H(t):=H(t)\left(\mu^{H} d t+\sum_{i=1}^{d} \xi_{i} d W_{i}(t)\right)
$$

where $\mu^{H}=r+\nu_{0}$ and $\xi_{i}(t)=\sigma_{i}\left(t, T_{0}\right)$, with $\nu_{0}=\sum_{i=1}^{d} \xi_{i}(t) \gamma_{i}(t)$.

The portfolio of assets $V(t)$ is given by the equation

$$
d V^{\pi}(t)=V^{\pi}(t)\left(\mu^{V} d t+\sum_{i=1}^{d} \theta_{i}^{V} d W_{i}(t)\right)
$$

where $\mu^{V}=\sum_{j \in K} \pi_{j} \mu_{j}$ and $\theta_{i}^{V}=\sum_{j \in K} \pi_{j} \sigma_{i j}$.

The process $X(t)=H(t) / V^{\pi}(t)$ is described by a particularly simple evolution equation. As we know from the previous section this is the equation

$$
\frac{d X}{X}=\mu^{X} d t+\sum_{i=1}^{d} \theta_{i} d W_{i}, \text { with } \mu^{X}=\mu^{H}-\mu^{V}-\sum_{i=1}^{d} \theta_{i}^{V} \theta_{i}, \theta_{i}=\xi_{i}-\theta_{i}^{V} .
$$

But in the present case

$$
\mu^{H}-\mu^{V}=\mu^{H}-\sum_{j \in K} \pi_{j} \mu_{j}=\sum_{i=1}^{d} \gamma_{i}\left(\xi_{i}-\sum_{j \in K} \pi_{j} \sigma_{i j}\right) .
$$

Hence $\mu^{X}$ is independent of $r$. As a result the HJB equation depends only on $t$ and $x$ and has the form

$$
u_{t}+\inf _{\pi \in S^{\pi}}\left(x u_{x} \mu^{X}+\frac{1}{2} x^{2} u_{x x} \sum_{i=1}^{d} \theta_{i}^{2}\right)=0 .
$$


Factoring the value function as previously

$$
u(t, x)=g(x) q(t)
$$

and substituting this factorization into equation (31) we obtain

$$
q_{t}+\inf _{\pi \in S^{\pi}}\left(\alpha q \mu^{X}+\frac{\alpha(\alpha-1)}{2} q \sum_{i=1}^{d} \theta_{i}^{2}\right)=0 .
$$

From this equation we obtain the optimal investment strategy which is independent of the solution of equation (33)

$$
\pi_{j}=\frac{1}{1+\alpha} \sum_{h \in K} S_{j h}^{-1}\left(\nu_{h}+\alpha Z_{h}\right)
$$

where

$$
\begin{aligned}
& S_{j h}=\sum_{i=1}^{d} \sigma_{i j} \sigma_{i h}, \quad j, h \in K, \\
& Z_{h}=\sum_{i=1}^{d} \xi_{i} \sigma_{i h}, \quad h \in K, \\
& \nu_{h}=\sum_{i=1}^{d} \gamma_{i} \sigma_{i h}, \quad h \in K .
\end{aligned}
$$

It is seen from this expression that the optimal strategy is the sum of a component which is independent of the market price of risk and a component depending on this price. Let us observe that as the investor's risk aversion increases ( $\alpha$ goes to infinity) the wealth is concentrated in the part independent of the price of risk.

Substituting expression (34) into the HJB equation (33) we obtain

$$
q_{t}+\alpha q\left(\frac{\alpha+1}{2} \sum_{j \in K} \sum_{h \in K} \pi_{j} \pi_{h} S_{j h}-\sum_{j \in K} \pi_{j}\left(\nu_{j}+\alpha Z_{j}\right)+\nu_{0}+\frac{\alpha-1}{2} \sum_{i=1}^{d} \xi_{i}^{2}\right)=0 .
$$

Solving equation (35) we get the value function

$$
\begin{aligned}
& q(t)= \\
& \exp \left(-\frac{\alpha}{2} \int_{t}^{T}\left(\frac{1}{\alpha+1} \sum_{j \in K} \sum_{h \in K} S_{j h}^{-1}\left(\nu_{j}+\alpha Z_{j}\right)\left(\nu_{h}+\alpha Z_{h}\right)-2 \nu_{0}-(\alpha-1) \sum_{i=1}^{d} \xi_{i}^{2}\right) d s\right) .
\end{aligned}
$$

5. The portfolio problem for the liability maturing at the planning horizon. We present the particular case of a single liability maturing at the planning horizon, when the economic factors are reduced to the spot interest rate modeled by the Gaussian HJM model. This can be considered as a special case of the problem considered in the previous section. The fact that the liability matures at the planning horizon creates additional difficulties due to the lack of boundedness of the obtained optimal trading strategies. This problem has been considered by several authors (cf. [1], [4], [12]). The satisfactory solution has been found recently by Bielecki, Pliska and Yong [4] for a particular model 
of economic factors. We present here another solution of the same problem in a different setting.

In this setting the portfolio of liabilities $H(t)$ consists of the single bond with maturity $T$. Hence putting $X(t)=H(t) / V^{\pi}(t)$ and considering the optimization problem

$$
\min _{\pi \in S^{\pi}(t, x, r)} \mathbb{E}[g(X(T)) \mid X(t)=x, r(t)=r],
$$

we can forget about the evolution of $H(t)$ as we know for sure that $H(T)=1$. The process $X(t)$ is described by the evolution equation

$$
\frac{d X}{X}=\mu^{X} d t+\sum_{i=1}^{d} \theta_{i}^{V} d W_{i}
$$

where

$$
\mu^{X}=-r-\sum_{j \in K} \pi_{j} \nu_{j}+\sum_{i=1}^{d}\left(\theta_{i}^{V}\right)^{2}, \quad \nu_{h}=\sum_{i=1}^{d} \gamma_{i} \sigma_{i h} \quad \text { and } \quad \theta_{i}^{V}=\sum_{j \in K} \pi_{j} \sigma_{i j} .
$$

In contrast to the problem in the previous section, $\mu^{X}$ depends now on $r$ and we have to take into account equation (29). As a result the HJB equation is in 3 variables

$$
\begin{aligned}
& u_{t}+\inf _{\pi \in S^{\pi}}\left(x u_{x} \mu^{X}+u_{r} \mu^{r}+\frac{1}{2} x^{2} u_{x x} \sum_{i=1}^{d}\left(\theta_{i}^{V}\right)^{2}+\frac{1}{2} u_{r r} \sum_{i=1}^{d} b_{i}^{2}-x u_{r x} \sum_{i=1}^{d} b_{i} \theta_{i}^{V}\right)=0, \\
& u(T, x, r)=g(x) .
\end{aligned}
$$

Assuming

$$
u(t, x, r)=g(x) q(t, r)
$$

we obtain the following equation for $q$

$$
\begin{aligned}
& q_{t}+\inf _{\pi \in S^{\pi}}\left(\alpha q \mu^{X}+q_{r} \mu^{r}+\frac{\alpha(\alpha-1)}{2} q \sum_{i=1}^{d}\left(\theta_{i}^{V}\right)^{2}+\frac{1}{2} q_{r r} \sum_{i=1}^{d} b_{i}^{2}-\alpha q_{r} \sum_{i=1}^{d} b_{i} \theta_{i}^{V}\right)=0, \\
& q(T, r)=1 .
\end{aligned}
$$

The optimal investment strategy can be calculated formally to give

$$
\pi_{j}=\frac{1}{1+\alpha} \sum_{h \in K} S_{j h}^{-1}\left(\nu_{h}+\frac{q_{r}}{q} B_{h}\right)
$$

where

$$
\begin{aligned}
& S_{j h}=\sum_{i=1}^{d} \sigma_{i j} \sigma_{i h}, \quad j, h \in K, \\
& B_{h}=\sum_{i=1}^{d} b_{i} \sigma_{i h}, \quad h \in K .
\end{aligned}
$$

The optimal strategy can be written in the abbreviated form

$$
\pi_{j}=\pi_{j}^{0}+\frac{q_{r}}{q} \pi_{j}^{1}
$$


with

$$
\begin{aligned}
\pi_{j}^{0} & =\frac{1}{1+\alpha} \sum_{h \in K} S_{j h}^{-1} \nu_{h}, \\
\pi_{j}^{1} & =\frac{1}{1+\alpha} \sum_{h \in K} S_{j h}^{-1} B_{h} .
\end{aligned}
$$

Substituting expression (39) into the HJB equation we obtain

$$
q_{t}+\alpha q \bar{\mu}^{X}+q_{r} \mu^{r}+\frac{\alpha(\alpha-1)}{2} q \sum_{i=1}^{d}\left(\bar{\theta}_{i}^{V}\right)^{2}+\frac{1}{2} q_{r r} \sum_{i=1}^{d} b_{i}^{2}-\alpha q_{r} \sum_{i=1}^{d} b_{i} \bar{\theta}_{i}^{V}=0 .
$$

Here as previously the bar over $\theta_{i}^{V}$ and $\mu^{X}$ denotes the values of these parameters taken at the point of the optimal strategy. Using formula (40) we arrive at the following expressions

$$
\begin{aligned}
a_{X} & =-r-\sum_{j \in K} \pi_{j}^{0} \nu_{j}+\sum_{j \in K} \sum_{h \in K} \pi_{j}^{0} \pi_{h}^{0} S_{j h}, & b_{X} & =-\sum_{j \in K} \pi_{j}^{1} \nu_{j}+2 \sum_{j \in K} \sum_{h \in K} \pi_{j}^{0} \pi_{h}^{1} S_{j h}, \\
a_{\theta} & =\sum_{j \in K} \sum_{h \in K} \pi_{j}^{0} \pi_{h}^{0} S_{j h}, & b_{\theta} & =2 \sum_{j \in K} \sum_{h \in K} \pi_{j}^{0} \pi_{h}^{1} S_{j h}, \\
a_{b} & =\sum_{j \in K} \pi_{j}^{0} B_{j}, & b_{b} & =\sum_{j \in K} \pi_{j}^{1} B_{j}, \\
c & =\sum_{j \in K} \sum_{h \in K} \pi_{j m}^{1} \pi_{h n}^{1} S_{j h} . & &
\end{aligned}
$$

After rearrangements the HJB equation takes the form

$$
q_{t}+A_{0} q_{r r}+A_{1} \frac{q_{r}^{2}}{q}+A_{2} q_{r}+A_{3} q=0,
$$

where

$$
\begin{aligned}
& A_{0}=\frac{1}{2} \sum_{i=1}^{d} b_{i}^{2}, \\
& A_{1}=\frac{1}{2} \alpha(\alpha+1) c-\alpha b_{b}, \\
& A_{2}=\mu^{r}+\alpha b_{X}+\frac{1}{2} \alpha(\alpha-1) b_{\theta}-\alpha a_{b}, \\
& A_{3}=\alpha a_{X}+\frac{1}{2} \alpha(\alpha-1) a_{\theta} .
\end{aligned}
$$

Equation (42) has to be solved in the strip $r \geq 0,0 \leq t \leq T$ with the boundary and terminal conditions

$$
\begin{aligned}
& q(t, 0)=1, \\
& q(T, r)=1 .
\end{aligned}
$$

After making the substitution

$$
z=\ln q
$$

we obtain the equation

$$
z_{t}+A_{0} z_{r r}+\left(A_{0}+A_{1}\right) z_{r}^{2}+A_{2} z_{r}+A_{3}=0,
$$


with the boundary and terminal conditions

$$
\begin{aligned}
& z(t, 0)=0, \\
& z(T, r)=0 .
\end{aligned}
$$

To solve equation (44) with conditions (45) we use the the 1-dimensional version of Theorem 3.1. Then we arrive at the analog of Theorem 3.2.

TheOREM 5.1. Under Assumptions 2.2, 2.3, 3.1 and 3.2 there exists a unique solution to the terminal problem (44)-(45). This solution belongs to $H^{2+\beta, 1+\beta / 2}\left(\Omega_{T}\right)$, where $\Omega_{T}=$ $[0, R] \times[0, T]$, for every $R<\infty$.

Let us observe that the conclusion of Corollary 3.1 applies also in this case. Hence the solution of Theorem 5.1 leads to bounded investment strategies. This is formally different from the result of Bielecki, Pliska and Yong [4], who obtained unbounded trading strategies. There is however no contradiction between both results since in contrast to Bielecki, Pliska and Yong's approach our formulation of the problem excludes the degeneracy which was present in their approach.

\section{References}

[1] I. Bajeux-Besnainou, J. V. Jordan and R. Portait, Dynamic asset allocation for stocks, bonds, and cash, J. Business 76 (2003), 263-287.

[2] D. Becherer and M. Schweizer, Classical solutions to reaction-diffusion systems for hedging problems with interacting Ito and point processes, Ann. Appl. Probab. 15 (2005), 11111144 .

[3] T. P. Bielecki and S. R. Pliska, A risk sensitive intertemporal CAMP with application to fixed income management, IEEE Trans. Automat. Control. 49 (2004), 420-432.

[4] T. P. Bielecki, S. R. Pliska and J. Yong, Optimal investment decisions for a portfolio with a rolling horizon bond and a discount bond, J. Theor. Appl. Finance 8 (2005), 871-913.

[5] T. Björk, Interest rate theory, in: Financial Mathematics, W. J. Runggaldier (ed.), LNM 1656, Springer, 1997, 53-122.

[6] M. J. Brennan, E. S. Schwartz and R. Lagnado, Strategic asset allocation, J. Econ. Dynam. Control 21 (1997), 1377-1403.

[7] M. J. Brennan and Y. Xia, Stochastic interest rates and the bond-stock mix, Eur. Finance Rev. 4 (2000), 197-210.

[8] T. Browne, Beating a moving target: optimal portfolio strategies for outperforming a stochastic benchmark, Fin. Stoch. 3 (1999), 275-294.

[9] J. C. Cox and C. F. Huang, Optimal consumption and portfolio policies when asset prices follow a diffusion process, J. Econ. Theory 49 (1989), 33-83.

[10] J. C. Cox and C. F. Huang, A variational problem arising in financial economics, J. Math. Econ. 20 (1991), 465-487.

[11] P. Dai Pra, W. J. Runggaldier and M. Tolotti, Pathwise optimality for benchmark tracking, IEEE Trans. Automat. Control. 49 (2004), 386-395.

[12] G. Deelstra, M. Grasselli and P.-F. Koehl, Optimal investment strategies in CIR framework, J. Appl. Prob. 37 (2000), 936-946.

[13] D. Duffie and R. Kan, A yield-factor model of interest rates, Math. Finance 6 (1996), 379-406. 
[14] H. Föllmer and P. Leukert, Efficient hedging: cost versus shortfall risk, Fin. Stoch. 4 (2000), 117-146.

[15] I. Karatzas and E. Shreve, Methods of Mathematical Finance, Springer, 1998.

[16] R. Korn, Optimal Portfolios, World Scientific, 1997.

[17] J. Liu, Portfolio selection in stochastic environments, Stanford GSB working paper, 2005.

[18] O. A. Ladyzhenskaya, V. A. Solonnikov and N. N. Uraltseva, Linear and Quasilinear Equations of Parabolic Type, Amer. Math. Soc., 1968.

[19] R. C. Merton, Optimum consumption and portfolio rules in a continuous time model, J. Econ. Theory 3 (1971), 373-413.

[20] R. C. Merton, An intertemporal capital asset pricing model, Econometrica 41 (1973), 866-887.

[21] M. Rutkowski, Self-financing trading strategies for sliding, rolling-horizon and consol bonds, Math. Fin. 9 (1997), 361-385.

[22] C. Sørensen, Dynamic asset allocation and fixed income management, J. Fin. Quant. Anal. 34 (1999), 513-531.

[23] J. Yong and X. Y. Zhou, Stochastic Controls. Hamiltonian Systems and HJB Equations, Springer, 1999. 